\title{
Drug-induced nephrotoxicity
}

Gabriel Teixeira Montezuma Sales ${ }^{1}$

(i) Renato Demarchi Foresto ${ }^{2}$

1. Universidade Federal de São Paulo, São Paulo, SP, Brasil 2. Hospital do Rim, São Paulo, SP, Brasil

http://dx.doi.org/10.1590/1806-9282.66.S1.82

\section{SUMMARY}

Acute kidney injury is a very common diagnosis, present in up to 60\% of critical patients, and its third main cause is drug toxicity. Nephrotoxicity can be defined as any renal injury caused directly or indirectly by medications, with acute renal failure, tubulopathies, and glomerulopathies as common clinical presentations. Some examples of drugs commonly associated with the acute reduction of glomerular filtration rate are anti-inflammatories, antibiotics, such as vancomycin and aminoglycosides, and chemotherapeutic agents, such as cisplatin and methotrexate. Cases of tubulopathy are very common with amphotericin B, polymyxins, and tenofovir, and cases of glomerulopathies are common with VEGF inhibitors, bisphosphonates, and immunotherapy, and it is also common to have more than one clinical presentation related to a single agent. Early diagnosis is essential for the good evolution of the patient, with a reduction of renal exposure to the toxic agent, which requires knowing the risk factors and biomarkers. General measures such as correcting hydroelectrolytic disorders and hypovolemia, monitoring the serum level, avoiding combinations with the synergy of renal injury, and looking for similar options that are less toxic are the foundations for the treatment of complications that are still common and often preventable.

KEYWORDS: Drug-related side effects and adverse reactions. Acute kidney injury. Kidney tubules.

\section{INTRODUCTION}

Acute kidney injury (AKI) is a very common diagnosis in both hospital and pre-hospital environments and may reach $60 \%$ of patients hospitalized in an intensive therapy unit. There has been a growth in its incidence in recent decades, which can be explained by an increase in risk factors such as advanced age, chronic kidney disease (CKD), and diabetes mellitus. Epidemiological studies show that nephrotoxicity is the third most common cause of AKD (Acute Kidney Disease), which has become worst in recent decades due to the more frequent use of drugs with the potential to cause kidney damage, with studies showing a frequency up to $20 \%$ of nephrotoxic drug use in critical patients ${ }^{1}$.

Although safety studies are necessary for the release of new drugs, side effects are often detected only after they are put into the market, when they start being used by different populations on a global scale. The reason for such high rates of drug-induced kidney injury is the function of drug and metabolite excretion performed by the kidneys, with the consequent exposure of its structures with high energy need (glomeruli and tubules) to the high concentration of exogenous substances ${ }^{2.3}$.

Drug-induced nephrotoxicity is defined by the presence of any kidney injury caused directly or indirectly by medication. The presentation varies from an acute or chronic reduced glomerular filtration rate (GFR) to nephrotic syndrome and hydroelectrolytic disorders (HED) related, respectively, to glomerular and tubular damage. For epidemiological purposes, most studies only consider an increase in creatinine, hindering a precise analysis of the true magnitude of the problem¹.

The loss of GFR is a late marker of kidney injury, and other biomarkers are being researched to allow 
for an earlier intervention, which could improve the prognosis of these patients. Although there is no benefit proven by high-quality studies, there are some promising candidates:

- KIM - 1 (Kidney Injury Molecule - V1), adhesion molecule produced in the proximal convoluted tubule (PCT), which is increased in urinary concentration in situations of ischemia and drug toxicity, with accuracy demonstrated for cisplatin, gentamicin, and cyclosporine, in some cases with increase 48 hours after the introduction of the toxic agent and before the GFR reduction.

- Beta-2 microglobulin is a protein produced mainly by lymphocytes that increases its urine concentration in inflammatory diseases, including infections, and autoimmune diseases. It is filtered at the glomerulus, reabsorbed in the TCP, and considered a marker of tubular injury. A study on kidney transplantation showed an increase before the worsening of kidney function in patients with toxicity by calcineurin inhibitor $(\mathrm{CNi})$, with high accuracy to differentiate rejection.

- Clusterin, a protein involved in the process of apoptosis and antiapoptosis found in various organs, including the kidney. It is not filtered and, in the tubules, is produced in stress situations to prevent cell death. Its greater accuracy in the diagnosis of tubular damage has been demonstrated, when compared to creatinine, with the use of cisplatin, vancomycin, tacrolimus, and gentamicin. It presents as much early increase as KIM-1 and does not increase in patients with glomerular damage.

- Cystatin C, a protein produced by all nucleated cells and filtered freely. It is completely reabsorbed in the proximal tubule and classically used as a way of estimating the GFR in conditions under stable kidney function conditions, in which the creatinine clearance is less reliable, such as in cirrhotic patients. Studies on amphotericin B, polymyxin, vancomycin, and cisplatin have shown a better correlation with renal toxicity when compared to creatinine ${ }^{4}$.

\section{EPIDEMIOLOGY}

The AKI epidemiology varies according to the criteria used and the profile of the hospitals included in each study, but the proportion related to drug toxicity reaches $25 \%$ of the cases. Approximately $20 \%$ of the cases require renal replacement therapy, which is related to increased mortality, with rates of over $60 \%$ in developing countries ${ }^{1.5}$.
A common problem when investigating the main etiology of AKI is the absence of tests to define the predominant mechanism of the injury. In clinical practice, it is common to have patients that are septic, hypotensive, with signs of dehydration, and on medication that is possibly toxic, all common causes of kidney injury.

To suggest causality, some criteria are proposed ${ }^{6}$ :

- Exposure to the agent for at least 24 hours.

- Injury mechanism related to a drug compatible with the condition presented.

- Investigation of other causes of nephropathy with evolution or clinical manifestations that are not completely compatible.

Knowing the risk populations is essential for the prevention and early diagnosis of nephrotoxicity. The main risk factors are related to the reduction of the renal functional reserve (glomeruli without the ability to increase the filtration rate), a larger concentration of drugs on the tubules, and synergistic injury mechanisms. Some examples are drugs with vasoconstrictor effect associated with diuretics and drugs that compete for the transporter responsible for tubular secretion, increasing the cytoplasm concentration, such as in the combination of cisplatin and aminoglycoside ${ }^{7}$.

TABLE 1. RISK FACTORS RELATED TO NEPHROTOXICITY BY DRUGS

\begin{tabular}{l|l|l} 
Advanced age & Hypovolemia & Chronic kidney disease \\
\hline$>1$ nephrotoxic & Hypoalbuminemia & Cardiopathy \\
\hline $\begin{array}{l}\text { Genetic polymor- } \\
\text { phisms }\end{array}$ & Diabetes & Obesity \\
\hline High doses & Hypotension & \\
\hline
\end{tabular}

Renal toxicity can be divided into dose-dependent and idiosyncratic. In the first type, both the prevention and the treatment involve minimizing the duration and concentration of the drug, especially in situations associated with higher risk, such as the concomitant use of other nephrotoxic substances, renal ischemia, and patients with CKD. In the second type, there are few measures associated with the prevention and, in general, the treatment involves completely avoiding the drug. Examples of the first type are vancomycin, aminoglycosides, cisplatin, methotrexate, nonsteroidal anti-inflammatories, while the second are causes of acute interstitial nephritis, like proton pump inhibitors, beta-lactams, and some causes of thrombotic microangiopathy (TMA), such as $\mathrm{CNi}$. 
TABLE 2. COMBINATIONS OF DRUGS WITH

SYNERGISTIC NEPHROTOXICITY

Cyclosporine/NSAIDS + Diuretic/ACEI/ARB

Aminoglycoside + Cisplatin/Cephalothin

Vancomycin + Piperacillin-tazobactam

Cyclosporine + Simvastatin

Methotrexate + Penicillins/Salicylate/Sulfas

To facilitate the diagnostic approach, it is important to know the clinical manifestations that are characteristic of each nephrotoxic drug; some of the most common are acute and chronic reduction of GFR, tubulopathies, and glomerulopathies, which will be illustrated below. To make matters more complicated, several agents present mixed characteristics, and it is common to have non-oliguric AKI with signs of PCT injury. We will not include drugs whose mechanisms of renal injury are indirect since they are not considered as primarily nephrotoxic, although they may be a cause for AKI. Some examples are loop diuretics, which can lead to dehydration, and renin-angiotensin-aldosterone system (RAAS) inhibitors, which cause vasodilation of the efferent arteriole, reduced intraglomerular pressure, and consequent worsening of renal function in risk situations, such as in patients who are septic, dehydrated, or with renal artery stenosis.

\section{ACUTE RENAL DISEASE / ACUTE KIDNEY INJURY \\ Nonsteroidal anti-inflammatory drugs (NSAIDs)}

Medication widely used around the world, usually of easy access without prescription and considered one of the main causes of nephrotoxicity caused by drugs. The main risk factor is relative or absolute hypovolemia due to its action inhibiting the prostaglandins, causing vasoconstriction of the afferent arteriole. Examples of susceptible populations are patients with sepsis, decompensated congestive heart failure, cirrhosis, dehydration, or in use of agents that act on the renal hemodynamics (cyclosporine, iodinated contrast, RAAS inhibitors).

NSAIDS have the ability to cause injury in virtually any renal compartment; its main effects are described in table 3. Most renal side effects are observed on all subclasses; however, a recent study showed that the incidence of nephrotic syndrome could be more related to the use of the non-selective type, especially when for more than 15 days and up to 2 years after exposure to the drug. Preventive measures involve choosing other options for analgesia in patients with increased risk of AKI, maintaining it for the minimum time possible, and correcting hypovolemia when present $^{8.9}$.

\section{VANCOMYCIN}

A widely used antibiotics against gram-positive hospital bacteria, it is a cause of AKI in 5 to $15 \%$ of the patients, depending on different specific risk factors, such as daily dose $>4 \mathrm{~g}$, treatment time $>14$ days, and high serum concentration, although there are cases even at therapeutic level (15 to $20 \mathrm{mg} / \mathrm{L}$ ). Oral vancomycin, due to its low absorption, is not a cause of toxicity.

The injury mechanism is unclear, but some experimental studies suggest the induction of tubular ischemia due to oxidative stress. Recently, the formation of vancomycin cylinders with uromodulin has been demonstrated, especially with high plasma values, and with early increased creatinine when compared with other nephrotoxic drugs ${ }^{2}$. The association with other potentially nephrotoxic drugs is a well-determined risk factor, as well as in other cases. For reasons that are not well described, the combination with piperacillin-tazobactam causes a synergistic effect for renal injury, something unexpected due to the non-toxicity of the drug alone ${ }^{10}$.

The treatment of choice is changing the medication by others with a similar spectrum of action, such as linezolid and daptomycin. Some studies suggest that teicoplanin could have a better safety profile, despite being in the same class, but there is no consensus regarding that information. In cases which it is not possible to interrupt the use of the drug, the most effective preventive measure is to monitor serum levels. After the end of the treatment, renal function tends to return to baseline levels ${ }^{\mathbf{1 1}}$.

\section{AMINOGLYCOSIDES}

The emergence of multidrug-resistant gram-negative bacteria susceptible to this ancient class of antibiotics is responsible for its growing use in recent decades, despite the high incidence of renal toxicity. Drugs are filtered in the glomerulus, with partial resorption in the PCT, via a receiver named Megalin. Inside the tubular cell, there is a connection with membrane phospholipids, with loss of protein synthesis and reduction of the mitochondrial 
function and consequent cell death, which happens in a greater proportion with more cationic molecules, such as gentamicin.

Their more severe adverse effects are nephro and ototoxicity, with an incidence of up to 50\% of non-oliguric AKI in high-risk patients, often with EHD, such as hypokalemia and hypomagnesemia. Several factors influence the ability to cause damage, such as concomitant renal ischemia, high serum concentration, and dosage with multiple daily doses.

Due to the high prevalence of renal toxicity, the KDIGO (Kidney Disease Improving Global Outcomes) recommendation is to avoid aminoglycosides whenever there is another less toxic antimicrobial option, which unfortunately is not often possible. In these cases, specific measures to minimize the damage must be carried out, such as the correction of hypokalemia and hypomagnesemia before the infusion, short time of use ( $<10$ days, preferably), avoid use in hypovolemic patients, dosage one time per day, and dose adjusted according to the serum level. In the case of amikacin, the suggested concentration in the first hour is $>20$ $\mu \mathrm{g} / \mathrm{mL}$ and after $18-24 \mathrm{~h}<5$ to $8 \mu \mathrm{g} / \mathrm{mL}$. High valley values are related to nephrotoxicity incidence and must be corrected by increasing the intervals between infusions ${ }^{5}$.

After discontinuation of the drug, the patient can continue presenting worsening of renal function for days, due to the slow clearance mechanism of the medication in the tubular cell. Despite this, the prognosis is apparently good, with a return to baseline renal function in most cases after the medication has been interrupted. However, some authors suggest irreversible damage and hyperfiltration of the remaining glomeruli as the mechanism responsible for the apparent recovery of renal function ${ }^{12}$.

\section{CISPLATIN}

Platin derivates are old chemotherapeutic agents that remain widely used for the treatment of various cancers, such as those of the digestive tract and lungs. The risk of AKI was evaluated in a 6-year cohort that included over 800 patients, with an incidence of $34 \%$; however, there were no cases of progression to endstage kidney disease.

The damage is caused mainly in PCT, where the drug is internalized in the S3 segment through the OCT-2 (organic cationic transporter 2), and, in high concentrations, it causes DNA injury, with consequent cell death. Cisplatin also has a vasoconstrictor effect, and there have been rare reports of TMA cases. In addition to reducing the GFR, it also causes hypophosphatemia, glycosuria, and hypomagnesemia (the latter in $>40 \%$ of the cases). As prophylaxis in high-risk patients, it is recommended to reduce the dose of the medication, perform absolute or relative hypovolemia correction, and replacement the drug by carboplatin or oxaliplatin, when possible ${ }^{13.14}$.

\section{METHOTREXATE}

An immunosuppressant frequently used in the treatment of autoimmune diseases that is related to AKI in high doses $\left(>500 \mathrm{mg} / \mathrm{m}^{2}\right.$, only used in chemotherapy against hematological neoplasms and sarcomas), with an incidence of up to $12 \%$. There is the formation of crystals in the distal tubule, which causes local inflammation and necrosis through the formation of oxygen free radicals. The main risk factors are toxic drug interactions (sulfas, beta-lactams, salicylates), the dose used, the presence of ascites or pleural effusion, and the characteristics classically associated with nephrotoxicity.

Preventive measures include high doses of folinic acid and intravenous hydration, preferably with an isotonic bicarbonate solution, to correct possible hypovolemia and to alkalinize the urine; these measures are effective to reduce the medication crystallization. Some protocols suggest infusion up to $200 \mathrm{~mL} /$ hour with a total of up to 2 liters before initiating methotrexate, with a measurement of urinary $\mathrm{pH}$ every hour and extra infusions if the target of $>7.0$ could not be reached. The plasma concentration of the drug can be measured with specific targets depending on the protocol used (most suggests $<0.1 \mu \mathrm{mol} / \mathrm{L}$ ). In cases of injury that is already established, there is the option of glucarpidase, which acts with high efficiency in the enzymatic cleavage of the drug; another option is intermittent or extended high-flow hemodialysis, with special care regarding rebound in plasma concentration ${ }^{13.15}$.

\section{GLOMERULAR}

VEGF (vascular endothelial growth factor) Inhibitors

VEGF is produced by podocytes and is responsible for maintaining the proper functioning and integrity of the glomerular basement membrane. 
Inhibition can be direct through receptor inhibitor ligands (bevacizumab) or indirect with anti-angiogenic tyrosine kinase inhibitors (sorafenib and sunitinib). These are classes of chemotherapeutic agents widely used, especially in gynecological and pulmonary neoplasms.

Two of the main side effects are hypertension and proteinuria, with incidences respectively up to 22 and $72 \%$, with a dose-dependent effect. However, less than $5 \%$ of cases present nephrotic proteinuria. Renal biopsies have shown several findings, including TMA and collapsing glomerulopathy. Suspending the drug is the treatment of choice, but there are cases of proteinuria persistence even after suspension. Nephroprotection with RAAS blockers is then recommended.

\section{CHECKPOINT INHIBITORS}

Monoclonal antibodies called immunotherapy that act by increasing the $\mathrm{T}$ lymphocyte response against cancer cells. The main subclasses are the CTLA-4 (cytotoxic T-lymphocyte-associated protein) inhibitors, with ipilimumab as an example, and PD-1/PDL-1 (programmed cell death ligand), such as nivolumab and pembrolizumab. They are available for the treatment of multiple metastatic neoplasms, such as lung cancer and melanoma, with excellent results considering advanced staging.

The main side effects are of immune mechanism, and the most common renal manifestations involve both acute interstitial nephritis and glomerulonephritis with different histological patterns (DLM, crescentic, membrane proliferative, membranous). There have been reports of proteinuria and hypertension in $16 \%$ of the patients who used nivolumab and multiple agents with evolution to acute interstitial nephritis and a reported incidence of AKI of $2 \%$ among users of this class of medications.

Because of the various possible causes related to renal dysfunction in oncologic patients, the current recommendation is to perform a renal biopsy whenever there is doubt about the etiology and, in the presence of acute interstitial nephritis, the recommendation is to start prednisone $1 \mathrm{mg} / \mathrm{Kg}$ and maintain the medication in mild cases, suspending it in cases of stage KDIGO 2 or 3 of AKI. It is not recommended to resume the use of medication in cases of improvement when the initial involvement was considered severe due to the high rate of recurrence ${ }^{16}$.

\section{BISPHOSPHONATE}

Class of drugs used as first-line treatment for osteoporosis and hypercalcemia of malignancy that acts by inhibiting osteoclasts, with a consequent reduction in the reabsorption of the bone matrix. It is composed of several different agents, but regarding renal damage, the main ones involved are pamidronate and zoledronate.

The main clinical manifestation is proteinuria, and collapsing FSGS is the most typical anatomopathological finding. There have also been reports of AKI soon after the infusion, with a mechanism that likely involves the apoptosis of tubular cells, and this is reported mainly when the infusion is faster than recommended. Both clinical presentations are rare, and the prognosis is relatively good after discontinuation of medication ${ }^{17}$.

\section{TUBULOPATHY}

\section{Amphotericin B}

Medication used to treat various systemic mycoses with at least three presentations available on the market, deoxycholate, liposomal, and lipid complexes. The main difference between them is the degree of renal toxicity, with the first being the most toxic, with rates up to $80 \%$. The injury mechanism involves diffuse and direct tubular injury through the increase of permeability of the cell membrane, and ischemia that is induced by vasoconstriction of the afferent arteriole, which explains why hypotension, use of RAAS inhibitors, and low intravascular effective volume are important risk factors.

The most common clinical manifestations are hypokalemia, hypomagnesemia, urinary concentration defect, and metabolic acidosis, in addition to reduced GFR, and they begin, in general, after one week of medication use. In patients with a high risk of kidney toxicity who evolve with an increase in serum creatinine after the beginning of medication, it is recommended to change it for less toxic formulations or different classes of antifungal agents, particularly echinocandins and voriconazole. In cases in which there is no alternative to the use of amphotericin deoxycholate, it is recommended to increase the time of infusion, hydration pre-infusion (500 to $1000 \mathrm{~mL}$ ), and HED monitoring and correction as preventive measures for AKI. The use of amiloride and spironolactone showed good results in the prevention of hypokalemia. Although the recovery of renal function is common after some weeks of 
discontinuation of the drug, the incidence of severe acute renal dysfunction was $10 \%$ and was associated with an increased risk of mortality ${ }^{5}$.

\section{POLYMYXINS (COLISTIN)}

Class of antibiotics whose use made a comeback in recent decades due to the emergence of multidrug-resistant gram-negative bacteria, like aminoglycosides, which also has a high rate of nephrotoxicity, with an incidence of up to $60 \%$ of AKI, often associated with hypokalemia and hypomagnesemia. There are two agents in clinical use currently, polymyxins B and $\mathrm{E}$ (colistin), which present important differences in terms of pharmacokinetics, and a need for adjusting the dose based on renal function in the case of colistin. The injury mechanism involves increased permeability of the cell wall and consequent edema and lysis, particularly in PCT.

Regarding the difference in toxicity, there is controversy about whether there is a lower incidence of renal toxicity with polymyxin $\mathrm{B}$, and there is a prospective study that shows a lower incidence of the advanced stage of AKI, although with no difference in mortality ${ }^{18}$. Part of the difference may have occurred due to the difficulty in determining the optimum dose of medication according to the renal function. Despite the good renal prognosis after discontinuation of the drug, this is often not possible due to the importance of adequate treatment of the infection, in many cases with evolution to severe renal lesion requiring renal replacement therapy. There is no specific treatment for nephrotoxicity, so the recommendation is to monitor and correct HED and prioritize the treatment for the infection, with a tendency to correct the dose of colistin based on renal function, but not polymyxin $B^{8.19}$.

\section{TENOFOVIR}

Antiretroviral therapy classified as nucleotide reverse transcriptase inhibitor and with widespread use since the beginning of the century for the treatment of HIV and hepatitis B. It is an integral part of the first-line regimen in Brasil due to its good tolerance, low resistance induction, and favorable dosage. However, it is not free from side effects; proximal renal tubulopathy and bone dystrophy, in particular, have been well described. The drug is internalized in the PCT cell by OAT- 1 and 3 (organic anion transporter), where, in high concentrations, it inhibits essential functions associated with substance transport. The initial clinical manifestations are proteinuria, hypophosphatemia, and glycosuria, in some cases evolving to CKD.

The exponential increase of users of this medication, with the broadening of indication of HIV treatment and the emergence of pre and post-exposure prophylaxis (currently, all patients are treated, regardless of CD4 and frequent communication) has made its toxicity even more evident. Several authors have demonstrated more frequent loss of renal function in patients who were chronic users of tenofovir, both as prophylaxis and treatment, and even over relatively short periods (6 to 48 months). Some specific risk factors related to tubular damage are the duration of drug use, association with protease inhibitors, especially atazanavir and ritonavir, previous reduction of GFR, systemic arterial hypertension, and age $>50$ years.

It is recommended to frequently monitor the renal function and markers of tubular injury, such as glycosuria and phosphaturia. Brazilian guidelines for the treatment of HIV from 2018 advocate for the replacement of the drug in patients with a reduction of $25 \%$ of the baseline GFR or when $<60 \mathrm{~mL} / \mathrm{min} / 1.73 \mathrm{~m}^{2}$. Currently, the wide variety of classes against HIV allows the exchange without prejudice for the control of the disease. In addition, since 2015, there is a prodrug with much lower renal toxicity approved by the FDA (Food and Drug Administration) called TAF (tenofovir alafenamide), but it is not available in Brasil. In highrisk patients, one option is to suspend the medication depending on the progression of PCT lesion markers, even before the reduction of $\mathrm{GFR}^{20.21}$.

\section{CHRONIC KIDNEY DISEASE}

Calcineurin inhibitors

Class of immunosuppressive agent essential in the prevention of rejection in solid organ transplantation and in the treatment of various glomerulonephritis. It is represented by cyclosporine and tacrolimus. Several mechanisms associated with $\mathrm{CNi}$ can cause renal injury, among the main ones are vasoconstriction of the afferent arteriole, hypertension, TMA, and induction of tubule-interstitial fibrosis and atrophy, with some authors suggesting a more pronounced hemodynamic effect with cyclosporine. The clinical presentation is also quite varied and may cause loss of glomerular filtration rate both acutely, by acute renal ischemia, and progressively over the years, with arteriolar and interstitial injury. 
TABLE 3. CORRELATION BETWEEN CLINICAL SYNDROMES, INJURY MECHANISMS, AND MEDICATIONS.

\begin{tabular}{|c|c|c|}
\hline Medications & Syndromes & Injury mechanism \\
\hline NSAIDs & $\begin{array}{l}\text { AKI } \\
\text { CKD } \\
\text { Glomerulopathy }\end{array}$ & $\begin{array}{l}\text { Vasoconstriction AA / AIN } \\
\text { TAIF } \\
\text { MID / MG }\end{array}$ \\
\hline $\mathrm{CNi}$ & $\begin{array}{l}\text { AKI } \\
\text { CKD }\end{array}$ & $\begin{array}{l}\text { Vasoconstriction AA / TMA } \\
\text { TAIF }\end{array}$ \\
\hline $\begin{array}{l}\text { Aminoglycosides } \\
\text { Cisplatin }\end{array}$ & $\begin{array}{l}\text { Tubulopathy } \\
\text { AKI }\end{array}$ & $\begin{array}{l}\text { Proximal tubular injury } \\
\text { Diffuse tubular injury }\end{array}$ \\
\hline $\begin{array}{l}\text { Polymyxins } \\
\text { Amphotericin B }\end{array}$ & $\begin{array}{l}\text { Tubulopathy } \\
\text { AKI }\end{array}$ & $\begin{array}{l}\text { Diffuse tubular injury } \\
\text { Vasoconstriction AA }\end{array}$ \\
\hline Tenofovir & $\begin{array}{l}\text { Tubulopathy } \\
\text { CKD }\end{array}$ & $\begin{array}{l}\text { Proximal tubular injury } \\
\text { Chronic interstitial nephritis }\end{array}$ \\
\hline $\begin{array}{l}\text { VEGF inhibitors } \\
\text { Gemcitabine } \\
\text { ImTOR }\end{array}$ & $\begin{array}{l}\text { Glomerulopathy } \\
\text { AKI }\end{array}$ & $\begin{array}{l}\text { Podocyte injury } \\
\text { TMA }\end{array}$ \\
\hline $\begin{array}{l}\text { PPI } \\
\text { Beta-Lactams } \\
\text { Allopurinol } \\
\text { Any drug } \\
\end{array}$ & $A K I$ & AIN \\
\hline $\begin{array}{l}\text { Topiramate } \\
\text { Amoxicillin } \\
\text { PI } \\
\text { Acyclovir } \\
\text { Methotrexate }\end{array}$ & $\begin{array}{l}\text { Nephrolithiasis } \\
\text { AKI }\end{array}$ & $\begin{array}{l}\text { Formation of crystals with induction of forma- } \\
\text { tion of calculi and tubular toxicity }\end{array}$ \\
\hline $\begin{array}{l}\text { Pamidronate } \\
\text { Infliximab }\end{array}$ & Glomerulopathy & Collapsing FSGS \\
\hline
\end{tabular}

The prevalence of nephrotoxicity is quite varied and is directly related to the exposure time and high plasma concentrations. In patients with glomerulonephritis and kidney transplantation, there are many confounding factors that complicate the analysis of incidence, but in other solid organs, the prevalence of CKD reaches $30 \%$, with studies suggesting the use of $\mathrm{CNi}$ as one of the main factors associated with it.

For the treatment, it is recommended to monitor plasma concentrations closely, but the decision to continue using the medication depends on the disease. In glomerulopathies, it is feasible to try other options of immunosuppressants, but in transplantations, the risk of nephrotoxicity, so far, is considered lower than that of rejection, so changing the medication is only suggested in particular situations, such as in some cases of TMA. Scenarios that evolve with AKI mainly associated to the vasoconstrictor effect tend to be reversible, unlike the slow and gradual loss associated with chronic nephrotoxicity ${ }^{22}$.

\section{DISCUSSION}

The evolution in the treatment of severe diseases, such as cancer and infections, with the emergence of increasingly effective drugs with different mechanisms of action, is a reality in medicine today. However, these medications bring various side effects, new and old, and nephrotoxicity is one of the most common and with the greater morbidity.

The focus of the treatment is trying to minimize the harm that can be caused by renal toxicity. Thus, early recognition of the condition is essential. In addition to the biomarkers already described in this text, other measures are emerging to minimize this problem. The use of electronic systems that alert the nephrologist for evaluation when the laboratory detects an increase in serum creatinine, with or without prescription of nephrotoxic drugs, has been well studied recently. Although still controversial, there is a tendency toward improvement this mechanism 
is used due to early application of measures against nephrotoxicity, such as the correction of dose, minimization of non-essential medications or particularly toxic combinations, and correction of hypovolemia, in addition to the investigation of other causes for renal disease ${ }^{23}$.

Another point that is being well studied is pharmacogenetics. It is known that some patients have a genetic predisposition to certain side effects related to the medication. One of the mechanisms already known involve transporters of drugs present in the PCT, with OAC and MRP (multidrug resistance protein transporter) mutations and proven involvement in more serious cell damage. This information can be useful when deciding to prescribe potentially toxic medications and the best way to follow-up these patients. In addition, in the future, they may become specific therapeutic targets, with the creation of medications with structures that allow lower concentration in the tubular cell, as is the case of tenofovir alafenamide ${ }^{2}$.

Since, so far, technology has not created perfect drugs exempt from adverse effects, the priority in cases of nephrotoxicity is based on the knowledge of risk populations, early diagnosis, and in general measures that can minimize the damage, such as correcting the dose of the medication based on the patient's renal function, something simple that, unfortunately, is not always carried out appropriately. Replacing nephrotoxic medications by others similar and less harmful, correcting the hypovolemia and HED, reducing the duration of treatment when possible, monitoring the serum levels, and avoiding particularly toxic combinations are cost-effective ways to significantly decrease the incidence of drug-induced renal injury, a common complication that can have great repercussion in patients treatment.

There is no conflict of interest in the present study. There was no external funding.

\section{Contribution of the authors:}

Gabriel Teixeira Montezuma Sales'; Renato Demarchi Foresto contributed substantially to the planning, drafting, and revision of this article.

\section{RESUMO}

A lesão renal aguda é um diagnóstico muito comum, presente em até $60 \%$ dos pacientes críticos, e sua terceira maior causa é a toxicidade de medicamentos. A nefrotoxicidade pode ser definida como qualquer lesão renal causada por medicamentos, direta ou indiretamente, tendo a insuficiência renal aguda, tubulopatias e glomerulopatias como apresentações clínicas comuns. Alguns exemplos de drogas comumente associadas à redução aguda da taxa de filtração glomerular são anti-inflamatórios, antibióticos, como a vancomicina e aminoglicosídeos, e agentes quimioterápicos, tais como cisplatina e metotrexato. Casos de tubulopatia são muito comuns com anfotericina B, polimixinas e tenofovir, já casos de glomerulopatias são comuns com inibidores de VEGF, bisfosfonatos e imunoterapia; também é comum ocorrer mais de uma apresentação clínica relacionada a um único agente. O diagnóstico precoce é essencial para a boa evolução do paciente, com a redução da exposição ao agente tóxico, o que requer conhecimento dos fatores de risco e biomarcadores. Medidas gerais, tais como a correção de distúrbios hidreletrolíticos e da hipovolemia, o monitoramento do nível sérico, evitar combinações com sinergia de lesão renal e procurar opções semelhantes e menos tóxicas são os alicerces do tratamento de complicações que são comuns e, muitas vezes, evitáveis.

PALAVRAS CHAVE: Efeitos colaterais e reações adversas relacionados a medicamentos. Lesão renal aguda. Túbulos renais.

\section{REFERENCES}

1. Kane-Gill SL, Goldstein SL. Drug-induced acute kidney injury: a focus on risk assessment for prevention. Crit Care Clin. 2015;31(4):675-84.

2. Perazella MA. Pharmacology behind common drug nephrotoxicities. Clin I Am Soc Nephrol. 2018;13(12):1897-908.

3. Lote CI, Harper L, Savage CO. Mechanisms of acute renal failure. Br J Anaesth. 1996;77(1):82-9.

4. Griffin BR, Faubel S, Edelstein CL. Biomarkers of drug-induced kidney toxicity. Ther Drug Monit. 2019;41(2):213-26.

5. KDIGO. Clinical practice guideline for acute kidney injury. Kidney Inter Suppl. 2012;2(1):1-138.

6. Awdishu L, Mehta RL. The 6R's of drug induced nephrotoxicity. BMC Nephrol. 2017;18(1):124.

7. Perazella MA. Renal vulnerability to drug toxicity. Clin | Am Soc Nephrol. 2009;4(7):1275-83.

8. Caires RA, Silva VT, Burdmann E, Coelho FO, Costalonga EC. Drug-induced acute kidney injury. In: Ronco C, Bellomo R, Kellum IA, Ricci Z, eds. Critical care nephrology. $3^{\text {rd }}$ ed. Philadelphia: Elsevier; 2019. p.214-21.
9. Bakhriansyah M, Souverein PC, van den Hoogen MWF, de Boer A, Klungel $\mathrm{OH}$. Risk of nephrotic syndrome for non-steroidal anti-inflammatory drug users. Clin J Am Soc Nephrol. 2019;14(9):1355-62.

10. Balci C, Uzun Ö, Arici M, Hayran SA, Yüce D, Ünal S. Nephrotoxicity of piperacillin-tazobactam combined with vancomycin: should it be a concern? Int J Antimicrob Agents. 2018;52(2):180-4.

11. Mergenhagen KA, Borton AR. Vancomycin nephrotoxicity: a review. J Pharm Pract. 2014;27(6):545-53.

12. Molitoris BA. Manifestations of and risk factors for aminoglycoside nephrotoxicity. UpToDate. 2017. [cited 2019 Sep 5]. Available from: https://www.uptodate.com/contents/ manifestations-of-and-risk-factors-for-aminoglycoside-nephrotoxicity

13. Malyszko J, Kozlowska K, Kozlowski L, Malyszko J. Nephrotoxicity of anticancer treatment. Nephrol Dial Transplant. 2017;32(6):924-36.

14. Latcha S, Jaimes EA, Patil S, Glezerman IG, Mehta S, Flombaum CD. Longterm renal outcomes after cisplatin treatment. Clin | Am Soc Nephrol. 2016;11(7):1173-9. 
15. Howard SC, McCormick J, Pui CH, Buddington RK, Harvey RD. Preventing and managing toxicities of high-dose methotrexate. Oncologist. 2016;21(12):1471-82

16. Perazella MA, Sprangers B. AKI in patients receiving immune checkpoint inhibitors. Clin J Am Soc Nephrol. 2019;14(7):1077-9.

17. Jia N, Cormack FC, Xie B, Shiue Z, Najafian B, Gralow JR. Collapsing focal segmental glomerulosclerosis following long-term treatment with ora ibandronate: case report and review of literature. BMC Cancer. 2015;15:535.

18. Rigatto MH, Oliveira MS, Perdigão-Neto LV, Levin AS, Carrilho CM, Tanita MT, et al. Multicenter prospective cohort study of renal failure in patients treated with colistin versus polymyxin B. Antimicrob Agents Chemother. 2016;60(4):2443-9.
19. Zavascki AP, Nation RL. Nephrotoxicity of polymyxins: is there any difference between colistimethate and polymyxin B? Antimicrob Agents Chemother. 2017;61(3). doi: 10.1128/AAC.02319-16.

20. Brasil. Ministério da Saúde. Secretaria de Vigilância em Saúde. Departamento de DST Aids e Hepatites Virais. Protocolo clínico e diretrizes terapêuticas para manejo da infecção pelo HIV em adultos. Brasília: Ministério da Saúde; 2018

21. Ray AS, Fordyce MW, Hitchcock MJ. Tenofovir alafenamide: a novel prodrug of tenofovir for the treatment of human immunodeficiency virus. Antiviral Res. 2016;125:63-70.

22. Issa N, Kukla A, Ibrahim HN. Calcineurin inhibitor nephrotoxicity: a review and perspective of the evidence. AM J Nephrol. 2013;37(6):602-12.

23. Goldstein SL. Nephrotoxicities. F1000Res. 2017;6:55. 